\title{
Evaluation of the Effect of Platelet Mediators to Increase the Skin's Collagen; A Randomized Clinical Trial
}

\author{
Hamidollah Afrasiabian ${ }^{1,2}$, Mitra Nourbakhsh ${ }^{3} \&$ Majid Sadeghizadeh ${ }^{4}$ \\ ${ }^{1}$ Member of the International Society for Complementary Medicine Research (ISCMR) \\ ${ }^{2}$ Ibn Sina research institute, Kargar Shomali Str, Enghelab Sqr, Tehran, Ian \\ ${ }^{3}$ Department of Biochemistry, Faculty of Medicine, Iran University of Medical Sciences, Tehran, Iran \\ ${ }^{4}$ Department of Genetics, Faculty of Biological Sciences, Tarbiat Modares University, Tehran Iran \\ Correspondence: Hamidollah Afrasiabian, Member of the International Society for Complementary Medicine \\ Research (ISCMR) and Ibn Sina research institute, unit21, floor2, nom1839, second str Kargar Shomali Str, \\ Enghelab Sqr, Tehran, Ian.
}

Received: May 21, 2017 Accepted: June 21, 2017 Online Published: June 30, 2017

doi:10.5539/gjhs.v9n9p52 URL: https://doi.org/10.5539/gjhs.v9n9p52

\begin{abstract}
Objective: The aim of this paper is to evaluate the effect of platelet-rich plasma mediators on the reconstruction of facial skin collagen by a platelet cream. Additionally, the cream was composited with plasma and some herbal compounds, which carried those factors through the skin. This method as a non-invasive skin will help the skin rejuvenation.
\end{abstract}

Materials and Methods: This study is a randomized clinical trial; patients referred to the clinic with positive cases from skin wrinkles along with $20 \mathrm{MHz}$ frequency ultrasound images of their skin faces. All individual enrolled in the study were randomly divided into two groups.

Group A 70cc cream including 60cc basic cream (emulsion of Lecithin and Eucerin) in addition of 5cc platelet-rich plasma and 5cc herbal extracted (Herdahelix, Musk and Genestein) to take a month received. Consequently, instructions for use of this cream is that $2.3 \mathrm{~g}$ of the cream every night to be affected.

Group B (controls) received 70cc of the cream involving 6cc basic cream (emulsion of Lecithin and Eucerin) along with $10 \mathrm{cc}$ placebo (glycerin) that every night $2.3 \mathrm{~g}$ of the cream to be applied on the face.

After a month increased the amount of facial skin collagen in each group was calculated and compared by ultrasound $20 \mathrm{MHz}$. In this project compare numerically and in terms of the amount of reflected energy (RE) was performed. In addition, the idea of using plant extracts and platelet mediators to penetrate into the skin was derived from Iranian traditional medicine.

Findings: Statistical analysis of the data collection was done by SPSS 18 software. Further, demographic characteristics including age, sex, occupation, socioeconomic status and education level had no effect on the response to treatment $(\mathrm{P}=0.221)$, but demographic indicator of age on response to treatment was effective $(\mathrm{P}=0.021)$.

Respond to the treatment (increase the amount of collagen in the skin) in group A and group B equal to $72.91 \%$ and $12.5 \%$ respectively. In group A with group B were significant differences in response to treatment, $\mathrm{P}=0.000$. The only side effect occurred in group A was mild irritation including redness and mild itching, which happened in 2 patients.

Conclusion: Employee non-invasive method of platelet cream rebuilds collagen in the skin effectively.

Keywords: Skin collagen reconstruction, platelet cream, Iranian traditional medicine, platelet rich plasma, numerical evaluation, skin collagen

\section{Introduction}

One of the most important materials used in cosmetics and people who suffer from infections or treatment actions due to drug deliveries and destruction their skin collagen tissue is the reconstruction skin cream or skin remodeling methods. 
Help skin regeneration and rejuvenation of the skin by increasing the production of collagen, one of the most important issues in medicine today is cosmetic and medical skin. Especially the skin of patients has been suffered as a result of infection, blood disorder, or treatment of tissue damage, also for those interested in the use of tonic cosmetics for skin rejuvenation. The main indicator of reconstruction and skin rejuvenation is increases the amount of collagen in the dermis layer (Moody, McCarthy, \& Hruza, 2003). There are two general methods for increasing the amount of collagen in the skin: invasive and non-invasive methods. Currently, platelet-rich plasma (PRP) injection method into the skin is one of effective invasive methods (Wroblewski, Mejia, \& Wright, 2010). As in this method patients have faced to symptoms such as swelling, allergic reaction, infection, cellulites or other minor surgical complications. Therefore, a large number of patients are skeptical during referred to the skin clinic for usage this method.

Recently, non-invasive laser is one of new methods that increase skin collagen, which is named as "non-invasive laser procedure" (Trelles, Allones, Levy, Calderhead, \& Moreno - Arias, 2004). But what is important in the assessment and calculation method of improving skin tone or collagen.

Until the last few years, the observer skin was based by taking image with medical report in diagnosis as well as treatment procedures. This method is qualitative and it will not be valid (Kono et al., 2007). Quantitative assessment increase collagen by measuring the energy reflected from the skin has solved this problem, which will be discussed in the materials and methods section.

The other thing, we will face in this study, the challenge of drug penetration to the skin. The project aimed to choice an uncomplicated method for making collagen.

In recent years, modern medicine through the skin for therapeutic purposes has been proposed. In 2008 and 2016 articles in this regard were published in Europe, two points in this article will draw attention. First is remove the keratin layer of the skin, another is the employed of nano-ingredients in those researches (Roberts, Pellett, \& Elizabeth Cross, 2002).

Dermal absorption mechanism will take three important steps, local attract the same spot of skin, regional integration that occurs in the tissue adjacent, and finally systemic absorption, which aims to deliver drugs to the bloodstream via the skin (Moghimi, Williams, \& Barry, 1998). An interesting point is that in ancient medicine enter the drug into the body through the skin is great interest. In traditional medicine in the field of pharmacy, one part is allocated to ointments that the skin drugs and has systemic absorption. About a thousand years ago Ibn Sina one of the Iranian scientists in field of traditional medicine said "drugs have two part as soft and thick segment, the soft may penetrate into the skin" (Abu-Asab, Amri, \& Micozzi, 2013).

Particularly, in ancient medicine the musk has been proposed as an agent to increase the absorption of drugs, musk fragrance substance that is extracted from male deer navel and the best of its kind in China is being produced (Pooya, 2016). In modern medicine, the use of Genistein (SMH, 2008) and the roots of seaweed (Fucus vesiculous) has been raised recently (Fujimura et al., 2001).

In traditional pharmacology some drugs well-known as laze, this kind of drugs are capable to penetrate into the skin for other drugs without harm tor skin tissue, function as well as its structure (Altmeyer \& El-Gammal, 1992).

This study, we have used the combination of modern medicine and ideas of the ancient Iranian medicine simultaneously. Mediator or intermediates for the production of platelets include a variety of compounds like diverse granules, calcium ions, histamine, serotonin, dopamine, cytokines and fibroblastic growing factor (FGF). All mentioned compounds was blended with a triplet herbal extracted involving herdahelix, musk and

genistein. In particular, those materials make increasing amount of drug accumulation into the skin, and also genistein mimic the function of mRNA and stimulating fibroblasts as well. How to evaluate a little research comes in detail within the part of materials and methods.

\section{Materials and Methods}

This research was a randomized clinical trial conducted under the supervision and cooperation of the professors of Iran University of Medical Sciences and Tarbiat Modares University in Iran between May 15 and September 30, in 2016. Patients were referred to clinic of the Ibn Sina dermatology in Tehran, Iran. Inclusion criteria include parameters such as wrinkles, dermal regeneration, and tonicity of the skin that patients referred to the treatment center to address. Ultrasound image of the facial skin was taken from all individuals and it's as was used the basis for study and for comparison purposes. 146 patients were referred to the clinic during the course of the study; the inclusion criteria included the symptoms of rinkle and ultrasound image of face skin.

Exclusion criteria were included skin allergy, corticosteroids, chronic skin diseases, and pregnancy, cancer, skin 
and anticoagulation drugs, and also, those who used tonic cosmetic creams during the past month. Therefore, from total individuals that participated in this study 40 people were excluded on the basis of exclusion criteria, and 106 people remained. The population of this study were based beyond $95 \%$ confidence $(\alpha=0.05)$ and then randomly divided into two groups.

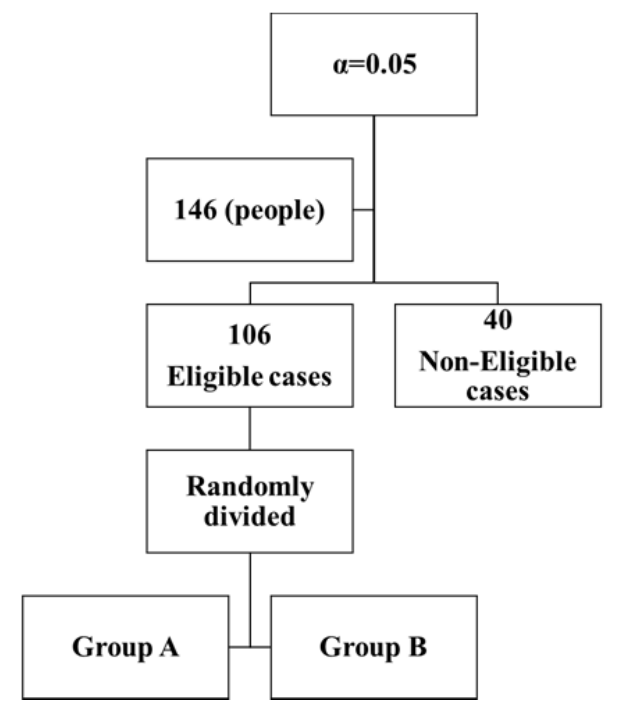

Figure 1. Demographic flowchart of clinical trial platelet cream along with inclusion and exclusion criteria

\subsection{Platelet Cream Preparation Method}

For group A the platelet cream was prepared including emulsion of Lecithin and Eucerin as basic cream (this cream purchased from Farabi Pharmaceutical Company of Iran). $10 \mathrm{cc}$ of blood was taken from each patient, then placed in a centrifuge machine at a speed of $1200 \mathrm{rpm}$ for 15 minutes (the centrifuge tube contains an anticoagulant). Finally, after completion of the plasma centrifuge, plasma plate was added to the cream base. At first, the amount of plasma solution is $5 \mathrm{cc}$. Then $5 \mathrm{cc}$ of herbal extracts from herdahelix, musk and genistein were added. Finally, the total creams of group A were $70 \mathrm{cc}$.

For group B $60 \mathrm{cc}$ of basic cream group A along with 10cc glycerin was added as placebo. Consequently, $70 \mathrm{cc}$ cream including placebo submitted to group B people. The cream was delivered to everyone in the two groups for one month's use. All people were advised to wash their face 2 hours before bed, rub it with a relatively rough texture, then dry the face. Then rub $2.3 \mathrm{~g}$ of the cream into the face skin slowly, also for each person, certain dishes with a capacity of $2.3 \mathrm{~g}$ of cream were prepared. In this study, the numerical comparison of the difference in skin tissue collagenization in two groups was very important. For the measurement of collagen before and after using the cream, ultrasound images of $20 \mathrm{MHz}$ frequency were used. Therefore, the B-mode scanner and the DUB-USB-Luneburg Germany devices were used in current project. Sonographic images of both groups were obtained before and after the use of platelet and placebo creams. The reflection of the frequency transmitted to the skin tissue appears as an increase in the amount of brightness in the skin (Fleming, 2000). Whatever collagen content per unit volume, collagen fibers are seen in bright or gray stripes. But the basis for comparing the tissue repair of the skin should be expressed in numerical units. In this image, the reflection energy of ultrasound radiation was evaluated based on the base ROI, to transform into a meaningful and numerical physical quantity; the radius energy square was expressed as the numerical quantity as shown in equation. Finally, a comparison was made by converting the numerical values of these values.

$$
\left[\mathrm{RE}=(\mathrm{RIO})^{2}\right]
$$

\subsection{Statistical Analysis}

The statistical analysis was used by SPSS18 software (SPSS Inc, Chicago, IL, USA) on numerical data concerning the software interpretation of reflected ultrasound waves.

\section{Results}

The results in the various aspects include; there is no significant correlation between demographic conditions of the participants and the amount of collagen in the skin through numerical evaluation of $\mathrm{RE}(\mathrm{P}=0.267)$. Since the demographic conditions in this research include age, sex, occupation, economic status and literacy level, hence the 
age demographic index had a significant effect on response to treatment with $\mathrm{P}=0.021$. Moreover, the effective factor in responding to treatment is the amount of skin collagen compression; the better collagen production is in the skin with a higher collagen density. Based on the calculations performed in this study, the minimum and maximum of the RE value or the reflectance energy square was 10 and 25 respectively. [RE $=10-25]$. But the amount of change in either the production or increase of collagen in the worst and best case was obtained 1 and 7 respectively. Noticeably, only one person in group A had the highest increase in collagen content. In group A, 2 patients left the study due to an allergic reaction, and 3 individuals did not continue the study. As in group B, 5 patients did not go on the study, so just 48 patients have remained in each group for completing the research.

In group A (intervention group), 13 patients did not respond to the use of cream. In other words, we had 27.03 percent negative or no response, while 35 patients responded to the intervention, with a positive therapeutic response of $72.91 \%$.

In group B (placebo group) no significant change was observed, where in 48 people, only 6 individuals responded, which was also very minor $[\mathrm{RE}=1-3]$. The success rate of placebo was only $12.5 \%$, with a little collagenization, but compared with group $\mathrm{A}$ with $\mathrm{P}=0.000$ was significantly lower.

The overall chart of the changes and the calculation of $\mathrm{P}_{\mathrm{value}}$ and its comparison in the group are discussed in more detail below.

Table 1. Shows the RE and $p$-value changes for groups A and B during the 30-days study.

\begin{tabular}{lllll}
\hline Group & Success $\%$ & RE Pre(mean) & RE Post(mean) & $P_{\text {value }}$ \\
\hline A & 72.91 & $18.65 \pm 3.31$ & $20.71 \pm 3.66$ & 0.00 \\
B & 12.5 & $18.98 \pm 3.35$ & $19.17 \pm 3.41$ & 1.00 \\
\hline
\end{tabular}

The following chart indicates the success rate of increase in collagen in column before and after using the cream.

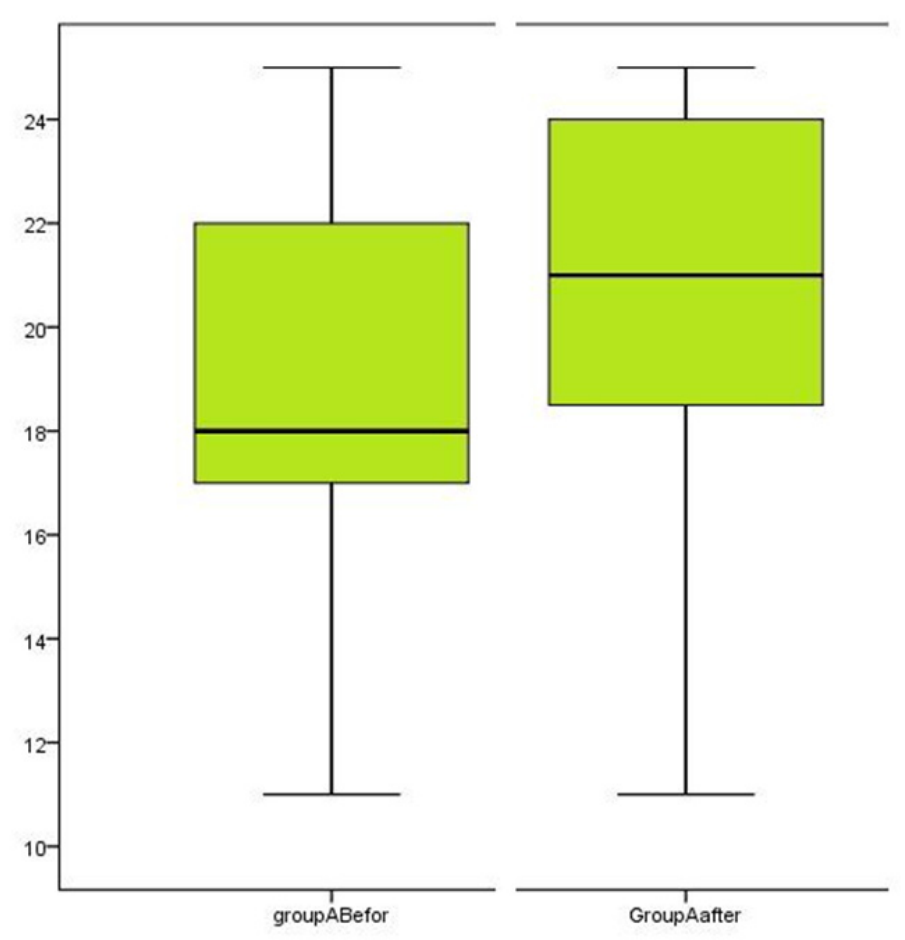

Figure 2. Changes of collagen in the skin before and after treatment based on the amount of reflected energy (RE)

\subsection{Side Effects}

As mentioned in the text above, in group A, only 2 patients showed allergic reactions in the form of redness and 
mild itching, which were excluded and therapeutic measures were taken, yet in group B there was no specific symptom. Also no systemic complications were observed for any of the two groups.

\section{Discussion}

In this study 106 patients as the population of study were participated to reconstruction of skin collagen and increasing of skin tissue toniste.

Based on ultrasound technology, images of $20 \mathrm{MHz}$ frequency were obtained from all individuals before the start of the study, and were reported numerically based on the reflectance energy square $\left(R^{2}\right)$. The participants randomly divided into two groups as the intervention group (A) and the control or placebo group (B). At the end of this study and after statistical analysis, the response rate was $72.91 \%$ in group A.

The motivation for choosing this study was to regeneration the skin with the employed of dermal penetration systems using complementary medicine. This is a new step in assessing the effect of skin medications on improving the tone of the skin of the human body.

There are two creative tips in this study, the first is the problem of non-invasive skin penetration, if successful, and a way to treat systemic skin problems is opened up to us. Thus, this success is due to traditional and complementary medicine, but it must be laboratory-tested, controlled and accurate.

In this study, we tried to discover a new way by examining the effect of treatment of skin problems through reflection energy and proving it numerically.

The second point is the beginning of a pathway to assess the claims of complementary and traditional medicine. One of the most important current medical science questions, and in particular the topics of the World Health Organization (WHO), is access to precise methods for evaluating and investigating allegations of traditional and complementary medicine. A detailed and standard statistical analysis is very promising in this regard. The role of placebo or induction in this study is interesting, but as it is seen, the results obtained in the control group occur partially. Traditional and alternative medicine seems to be a great source of experiences that will provide the basis for future research ideas in field of modern medical science.

In conclusion, we can say that the production of a tonic and organic cream is very important from the economic point of view in the cosmetics industry, and it will be economically and socially positive.

\section{Acknowledgments}

The authors would to acknowledge from academics staffs of Iran University of Medical Sciences and Tarbiat Modares University, Tehran Iran that had utmost cooperation and cooperation with me. Furthermore, I am going to have so many thanks warmly from all participated individuals, financial supporter of this project and also whole members of my clinic service.

\section{Competing Interests Statement}

The authors declare that there are no competing or potential conflicts of interest.

\section{References}

Abu-Asab, M., Amri, H., \& Micozzi, M. S. (2013). Avicenna's medicine: a new translation of the 11th-century canon with practical applications for integrative health care. Inner Traditions/Bear \& Co.

Altmeyer, P., \& El-Gammal, S. (1992). Ultrasound in dermatology. Springer.

Fleming, T. (2000). PDR for herbal medicines. Montvale, NJ: Medical Economics Company. vi.

Fujimura, T., Tsukahara, K., Moriwaki, S., Kitahara, T., Sano, T., \& Takema, Y. (2001). Treatment of human skin with an extract of Fucus vesiculosus changes its thickness and mechanical properties. Journal of cosmetic science, 53(1), 1-9.

Kono, T., Groff, W. F., Sakurai, H., Takeuchi, M., Yamaki, T., Soejima, K., \& Nozaki, M. (2007). Comparison study of intense pulsed light versus a long-pulse pulsed dye laser in the treatment of facial skin rejuvenation. Annals of plastic surgery, 59(5), 479-483. https://doi.org/10.1097/SAP.0b013e3180327943

Moghimi, H. R., Williams, A. C., \& Barry, B. W. (1998). Enhancement by Terpenes of 5 - Fluorouracil Permeation through the Stratum Corneum: Model Solvent Approach. Journal of pharmacy and pharmacology, 50(9), 955-964. https://doi.org/10.1111/j.2042-7158.1998.tb06909.x

Moody, B. R., McCarthy, J. E., \& Hruza, G. J. (2003). Collagen remodeling after 585 - nm pulsed dye laser irradiation: an ultrasonographic analysis. Dermatologic surgery, 29(10), 997-1000. 
https://doi.org/10.1097/00042728-200310000-00001

Pooya, H. K. (2016). Medicine and evolution process in ancient Persia. History of Medicine Journal (Quarterly), 2(3), 67-98.

Roberts, M. S., Pellett, M. A., \& Elizabeth Cross, S. (2002). Skin transport Dermatological and transdermal formulations (pp. 88-194): CRC Press.

SMH, A. (2008). Makhzan Al-Advieh.

Trelles, M. A., Allones, I., Levy, J. L., Calderhead, R. G., \& Moreno - Arias, G. A. (2004). Combined Nonablative Skin Rejuvenation with the 595 - and 1450 - nm Lasers. Dermatologic surgery, 30(10), 1292-1298.

Wroblewski, A. P., Mejia, H. A., \& Wright, V. J. (2010). Application of platelet-rich plasma to enhance tissue repair. Operative Techniques in Orthopaedics, 20(2), 98-105. https://doi.org/10.1053/j.oto.2009.10.006

\section{Copyrights}

Copyright for this article is retained by the author(s), with first publication rights granted to the journal.

This is an open-access article distributed under the terms and conditions of the Creative Commons Attribution license (http://creativecommons.org/licenses/by/4.0/). 